\title{
ANÁLISE EM CONDIÇÕES NÃO ISOTÉRMICAS DE UM AÇO INOXIDÁVEL SUPERDUPLEX ASTM AI 82 - F53 VIA DILATOMETRIA
}

\author{
Camila dos Santos Pinto ' \\ Gabrielle Cristine Lemos Duarte Freitas ' \\ Isabele Cristina Abreu de Sá ' \\ Gláucio Soares da Fonseca ' \\ Luciano Pessanha Moreira' \\ Paulo Rangel Rios '
}

\section{Resumo}

O aço ASTM AI 82 - F53, comercialmente SAF 2507, conhecido como superduplex, é frequentemente utilizado em indústrias offshore, de papel e celulose, devido a suas ótimas propriedades mecânicas e de resistência à corrosão, em função da estrutura balanceada de ferrita e austenita. Quando submetido a elevadas temperaturas, este balanço entre as fases pode ser comprometido e fases intermetálicas que degradam suas propriedades e limitam a sua utilização podem precipitar. A principal fase intermetálica é a fase sigma que nucleia e cresce no aço inoxidável e como consequência degrada as suas propriedades mecânicas e de resistência à corrosão. Os principais estudos na área simulam situações de envelhecimento, mantendo o material em um patamar isotérmico, para propiciar a formação desta fase e resfriamento rápido para posterior caracterização da fase sigma. Neste trabalho, as amostras sofreram ciclos térmicos (CT) de resfriamento sob taxas controladas em condições não isotérmicas em um simulador termomecânico Gleeble. A caracterização microestrutural após os CT foi realizada via microscopia ótica e eletrônica de varredura acoplada com detector de espectro de energia dispersiva. Como resultado, a ferrita se transforma em austenita secundária e fase sigma. O que é confirmado pela microscopia/EDS e pelos picos de temperaturas indicando a transformação de ferrita em fase sigma nas curvas dilatométricas.

Palavras-chave: Aço inoxidável superduplex; Transformações de fase; Transformação não isotérmica; Simulação Física, Dilatometria.

\section{ANALYSIS IN NON-ISOTHERMAL CONDITIONS OF SUPERDUPLEX STAINLESS STEEL ASTM AI 82 - F53 BY DILATOMETRY}

\begin{abstract}
The Steel ASTM AI 82 - F53, commercially SAF 2507, known as superduplex, is widely used in offshore, pulp and paper industries due to its excellent mechanical properties and corrosion resistance, depending on the balanced structure of ferrite and austenite. When subjected to high temperatures, this balance between phases can be compromised, and intermetallic phases that degrade their properties and limit their use may precipitate. The main intermetallic phase is the sigma phase that nucleates and grows in stainless steel and therefore degrades its mechanical properties and corrosion resistance. The main studies in the area simulate aging situations, keeping the material in an isothermal level, to provide the formation of this phase and rapid cooling for later characterization of the sigma phase. In this work, the samples were submitted to thermal cooling cycles (TC) under controlled rates in non-isothermal conditions in a Gleeble thermomechanical simulator. Microstructural characterization after TC was performed by optical microscopy and scanning electron microscopy coupled to a dispersive energy spectrum detector. As a result, the ferrite turns into secondary austenite and sigma phase. That is confirmed by microscopy/EDS and temperature peaks indicating the transformation of ferrite into sigma phase in the dilatometric curves. Keywords: Stainless steel superduplex; Phase transformations; Non-isothermal transformation; Physical simulation; Dilatometry.
\end{abstract} 'Programa de Pós-graduação em Engenharia Metalúrgica - PPGEM, Escola de Engenharia Industrial Metalúrgica de Volta Redonda - EEIMVR,
Universidade Federal Fluminense - UFF, Volta Redonda, RJ, Brasil. E-mail: camilapinto@id.uff.br

2I 76-I523 @ 2019 Associação Brasileira de Metalurgia, Materiais e Mineração. Published by ABM. This is an open access paper, published under the Creative Commons CC BY-NC-ND license (Attribution-NonCommercial-NoDerivs) - https://creativecommons.org/licenses/ by-nc-nd/4.0/. 


\section{INTRODUÇÃO}

Os aços inoxidáveis superduplex (AISD) combinam favoravelmente as propriedades dos aços inoxidáveis ferríticos e austeníticos. Eles possuem alta resistência mecânica, boa tenacidade, adequada resistência à corrosão em vários meios e excelente resistência à corrosão sob tensão. Estes aços são frequentemente utilizados em indústrias químicas e em aplicações offshore que requerem alta resistência à corrosão e excelente resistência mecânica [I - | |4]. O contínuo desenvolvimento dos AISD resulta em aços com composição química complexa, contendo considerável quantidade de elementos de liga. Esta substancial quantidade de elementos de liga presente nestes aços tem por objetivo melhorar as propriedades mecânicas e de resistência à corrosão. Como usual, os beneficios de tais adições invariavelmente vem com inevitáveis problemas. O principal problema é a instabilidade termodinâmica microestrutural $[7,15]$.

Como consequencia desta instabilidade microestrutural, durante processamento (tratamento térmico, soldagem) ou em uso, a precipitação de fases intermetálicas indesejáveis, tais como, chi, sigma $(\sigma)$, carbonetos e nitretos entre outras pode ocorrer [1-14]. A presença destas fases deletérias deteriora as propriedades mecânicas e de resistência à corrosão destes aços. A fase sigma se apresenta em um significativo maior volume quando se comparada as outras fases intermetálicas observadas nos trabalhos citados anteriormente em microestruturas duplex. Portanto, em muitos casos, é comum que as outras fases precipitadas nestes aços, sejam desconsideradas ou tem seu efeito combinado com a fase sigma nas análises das propriedades do material. Assim, a fase sigma é considerada a principal responsável pela degradação de tais propriedades nos AISD. Portanto é de grande importância entender as condições que levam a formação da fase sigma, com o objetivo de evitar sua formação durante processamento ou em uso e assim preservar as propriedades dos AISD.

A maioria dos estudos nesta área simulam situações de envelhecimento, mantendo o material em um patamar isotérmico, para propiciar a formação da fase sigma, e resfriamento rápido para posterior caracterização microestrutural $[1,3-5,8-10,13]$. Mas na prática, a maioria das transformações de fases, ocorre em condições não-isotérmicas. Mittemeijer e colaboradores [16], em um trabalho clássico, aplicaram um método para análise cinética de transformações no estado sólido em condições não isotérmicas para diferentes tipos de materiais. O ponto chave é encontrar a temperatura na qual ocorre a transformação de fase. Um dos métodos usados para determinar as temperaturas de transição de fases é o método dilatométrico, através do qual é possível monitorar as variações de comprimento de um corpo de prova em condições de resfriamento ou aquecimento. Assim, por meio de um método empregando a derivada da curva, é possível encontrar os pontos de inflexão na curva de expansão volumétrica versus temperatura ou tempo, sendo assim, uma forma de identificar indiretamente a fase transformada [16-19].

Poucos estudos caracterizam a formação da fase sigma em aços inoxidáveis duplex e superduplex em ciclos térmicos envolvendo taxas de aquecimento [19] e resfriamento [17, 18]. Portanto, neste trabalho é analisada a transformação de fase do aço inoxidável superduplex ASTM AI 82 - F53, chamado a partir deste momento neste trabalho de $F 53$, em condições não isotérmicas a diferentes taxas de resfriamento via análise térmica no simulador termomecânico Gleeble $3500^{\circledR}$. A caracterização microestrutural após os ciclos térmicos foi realizada via microscopia ótica (MO) e eletrônica de varredura (MEV) acoplada com detector de espectro de energia dispersiva (EDS). $O$ ensaio de microdureza Vickers também foi realizado para corroborar com as análises microscópicas.

\section{MATERIAL E MÉTODOS}

\section{I Material}

Para o presente estudo foi utilizado uma chapa de comprimento $L$ com dimensões iguais a $20 \mathrm{~mm}$ de largura e $10 \mathrm{~mm}$ de espessura. $O$ material consiste em um aço comercial inoxidável superduplex de alta liga comumente chamado de SAF 2507. Chamado no presente trabalho de F53. A composição química deste aço está descrita na Tabela I. Este material é do mesmo lote que foi utilizado em trabalhos anteriores[I,3]. A composição apresentada na Tabela I foi fornecida pela empresa.

Para a execução da simulação térmica no simulador termomecânico Gleeble $3500^{\circledR}$, os corpos de prova foram usinados conforme as dimensões especificadas na Figura I.

\subsection{Métodos}

\subsection{Testes dilatométricos em condições não isotérmicas}

Para a aplicação em resfriamento contínuo foram utilizados três corpos de prova que, foram aquecidos a taxa de $20^{\circ} \mathrm{C} / \mathrm{s}$ e homogeneizados a $1100^{\circ} \mathrm{C}$ durante $30 \mathrm{~min}$, onde se obtém de forma parcial as duas fases ferrita $(\alpha)$ e austenita $(\gamma)$ na matriz [ 17$]$. O resfriamento foi realizado em diferentes taxas $1,0^{\circ} \mathrm{C} / \mathrm{min}, 2,0^{\circ} \mathrm{C} / \mathrm{min}, 3,5^{\circ} \mathrm{C} / \mathrm{min}$ até $400^{\circ} \mathrm{C}$. Pois, segundo a literatura, as precipitações

Tabela I. Composição química do aço superduplex F53 (\% peso)

\begin{tabular}{ccccccccccc}
\hline $\boldsymbol{C}$ & $\boldsymbol{S i}$ & $\mathbf{M n}$ & $\boldsymbol{P}$ & $\boldsymbol{S}$ & $\boldsymbol{C r}$ & $\mathbf{N i}$ & $\mathbf{M o}$ & $\mathrm{Cu}$ & $\boldsymbol{N}$ \\
\hline 0,020 & 0,328 & 0,85 & 0,027 & 0,0009 & 24,89 & 6,82 & 3,72 & 0,156 & 0,278 \\
\hline
\end{tabular}



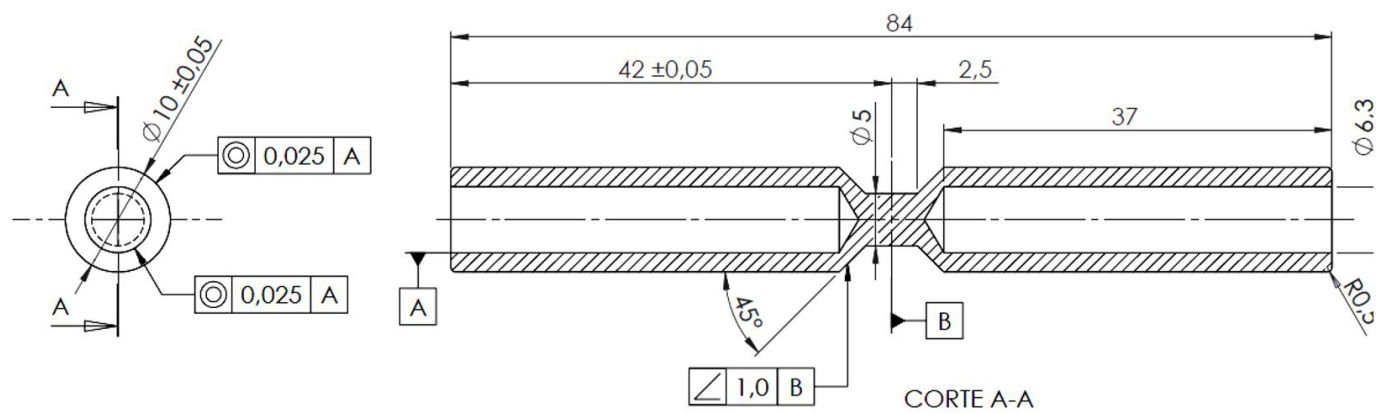

Figura I. Desenho técnico do corpo de prova utilizado para o simulador termomecânico Gleeble $3500^{\circledR}$.

da fase deletérias, tais como: chi, sigma, etc., neste tipo de aço ocorrem acima de $500^{\circ} \mathrm{C}$ [I-I4]. Mediante este entendimento, para os corpos de prova empregaram-se as taxas de resfriamento de $1100^{\circ} \mathrm{C}$ a $400^{\circ} \mathrm{C}$ e posteriormente a esta temperatura foram realizadas as têmperas. Através dos dados de ensaio são obtidas as curvas de dilatação $\left(\Delta d / d_{0}\right)$ versus temperatura $\left({ }^{\circ} \mathrm{C}\right)$, onde são observadas as temperaturas de pico de transformações de fases nos pontos onde a curva se desvia da linearidade (pontos de inflexão). Para determinar a temperatura exata, aplica-se o método da primeira derivada na curva de dilatação versus temperatura $[16,19]$.

\subsubsection{Caracterização microestrutural e ensaio de dureza}

Além das amostras com resfriamento controlado, descritas no item anterior, uma amostra foi aquecida em um forno Brasimet tipo $\mathrm{K} 250 \mathrm{~N} 2$ até $1100^{\circ} \mathrm{C}$ e permaneceu no forno por 30 min e depois foi resfriada em água. A microestrutura obtida desta amostra foi considerada a "Como Recebida (CR)", pois ela representa a microestrutura inicial antes das transformações de fase ocorridas nas amostras que passaram pelo resfriamento controlado na Gleeble.

A fim de revelar as microestruturas presentes, as amostras foram lixadas de 320 a 2500 mesh, depois realizou-se o polimento mecânico com pasta de diamante de $6 \mu, 3 \mu \mathrm{e} I \mu \mathrm{e}$ por último realizado o ataque químico Behara, composto por $20 \mathrm{ml}$ de ácido clorídrico $(\mathrm{HCl}), 80 \mathrm{ml}$ de água destilada $\left(\mathrm{H}_{2} \mathrm{O}\right)$ e, a cada $100 \mathrm{ml}$ desta solução estoque, adiciona-se $0,3 \mathrm{~g}$ de metabissulfito de potássio $\left(\mathrm{K}_{2} \mathrm{~S}_{2} \mathrm{O}_{5}\right)$, com tempo de imersão em torno de 30 à $45 \mathrm{~s}$. Com este ataque é possível diferenciar ferrita $(\alpha)$, austenita $(\gamma)$ e sigma $(\sigma)[1,3,8]$.

Após a etapa de preparação metalográfica, as micrografias foram obtidas com o auxílio do microscópio óptico Olympus Modelo BX5IM. Dez micrografias foram coletadas de cada amostra para realizar a quantificação de fases com o auxílio do software de domínio público Image J. A fração volumétrica das fases $\left(V_{v}\right)$ foi determinada, usando a relação padrão de metalografia quantitativa, igualando com a fração da área $\left(A_{A}\right)$ ocupada por determinada fase na microestrutura [20]. Os erros nas medidas foram menores que $10 \%$.
Posteriormente, as amostras foram analisadas em microscópio eletrônico de varredura EVO MA 10 acoplado com detector de espectro de energia dispersiva (EDS) para determinação da composição química das fases presentes. Foram escolhidos 5 pontos para cada fase para cada taxa de resfriamento e na amostra CR.

Com o intuito de verificar a relação entre microdureza e precipitação da fase sigma, foram realizadas 10 medidas de microdureza Vickers em cada amostra utilizando o durômetro HMV - 2 Shimadzu, com carga de 0,9807N $(0, I \mathrm{~kg})$, com um tempo de indentação de $15 \mathrm{~s}$. O coeficiente de determinação $\left(R^{2}\right)$ correspondente a uma relação linear entre a fração volumétrica de fase sigma e a dureza também foi avaliado.

\section{RESULTADOS E DISCUSSÃO}

\section{I Testes Dilatométricos}

A partir dos dados registrados, as curvas de variação dilatométrica e suas derivadas foram obtidas. A Figura 2 é um exemplo de curva dilatométrica obtida para a taxa de $1,0^{\circ} \mathrm{C} / \mathrm{min}$ junto com sua curva da primeira derivada, a qual é caracterizada pela presença de um pico negativo, indício de transformação, na faixa de temperatura de $700^{\circ} \mathrm{C}$ a $800^{\circ} \mathrm{C}$. Já a Figura 3, apresenta os picos negativos das curvas das primeiras derivadas referentes a cada taxa de resfriamento.

As temperaturas de transformação para as taxas de $3,5^{\circ} \mathrm{C} / \mathrm{min} ; 2,0^{\circ} \mathrm{C} / \mathrm{min}$ e $1,0^{\circ} \mathrm{C} / \mathrm{min}$ foram $762^{\circ} \mathrm{C}, 700^{\circ} \mathrm{Ce} 702^{\circ} \mathrm{C}$, respectivamente. Observa-se então que para taxas de resfriamento menores $\left(1,0\right.$ e $\left.2,0^{\circ} \mathrm{C} / \mathrm{min}\right)$, a transformação se dá praticamente na mesma temperatura (por volta de $700^{\circ} \mathrm{C}$ ), temperatura menor em comparação a taxa de $3,5^{\circ} \mathrm{C} / \mathrm{min}\left(762^{\circ} \mathrm{C}\right)$. Por meio das referências apresentadas neste trabalho [I-14], pode-se supor que nestas temperaturas ocorreu a precipitação de fase sigma. No entanto, como esta análise é feita de forma indireta, fez-se necessário o uso da microscopia eletrônica de varredura acoplada com detector de espectro de energia dispersiva (EDS) para validar tal afirmação. 


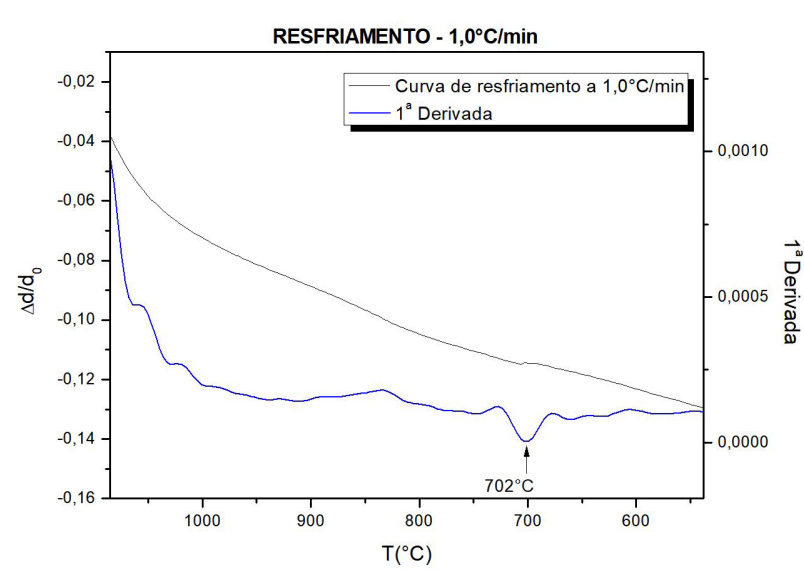

Figura 2. Curva de resfriamento e sua derivada referente à taxa de resfriamento $1,0^{\circ} \mathrm{C} / \mathrm{min}$; temperatura de transformação $-702^{\circ} \mathrm{C}$.

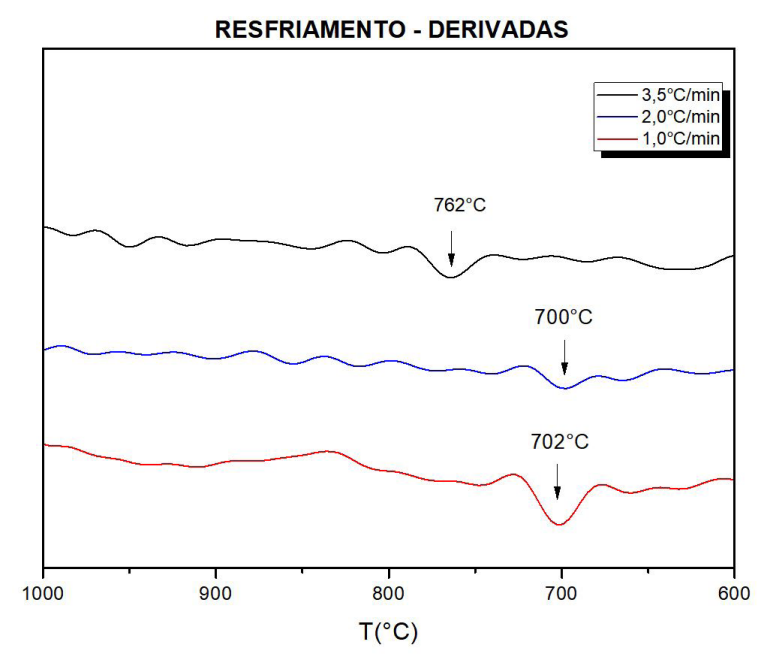

Figura 3. Curvas da primeira derivada para as taxas de resfriamento de: $3,5^{\circ} \mathrm{C} / \mathrm{min} ; 2,0^{\circ} \mathrm{C} / \mathrm{min}$ e $1,0^{\circ} \mathrm{C} / \mathrm{min}$ em função da temperatura.
Como pode ser notado na Figura 3, o pico de transformação encontrado decresceu à medida que ocorreu a redução das taxas $\left(1,0 / 2,0\right.$ para $\left.3,5^{\circ} \mathrm{C} / \mathrm{min}\right)$, ou seja, durante o resfriamento contínuo, o potencial termodinâmico ou maior superresfriamento para o início da transformação acompanhou o resultado para a taxa de resfriamento mais rápida.

\subsection{Caracterização Microestrutural}

A Figura 4 revela a microestrutura da amostra CR, com as fases ferrita e austenita presentes na matriz e, por se tratar de uma amostra inicial laminada, a morfologia dos grãos mesmo após a solubilização exibe a forma alongada. A porcentagem de ferrita obtida foi de $52 \pm 1 \%$ e de austenita $48 \pm 1 \%$. Logo o tratamento de homogeneização proposto foi eficaz em manter a matriz duplex.

Já na Figura 5 são apresentadas as micrografias obtidas após os resfriamentos controlados e exibem a formação de uma nova fase, possivelmente a fase sigma, que aparece com uma coloração cinza claro em forma de lamelas, originadas da ferrita, juntamente com possivelmente a austenita secundária $\left(\gamma_{2}\right)$. Para confirmar as fases presentes, foi realizada a determinação da composição química de cada fase através da técnica de EDS. Levando em consideração os elementos Cromo, Níquel e Molibdênio. A Tabela 2 apresenta a porcentagem média dos elementos em cada fase:

A Tabela 2, apresenta o resultado das análises por EDS das amostras referentes às taxas de resfriamento controlados e como recebida. Nota-se claramente que a fase sigma é mais rica em $\mathrm{Cr}$ e Mo que a austenita secundária. Em compensação a austenita secundária apresenta maior teor de Ni que a fase sigma. A precipitação da fase sigma ocorre principalmente a partir da decomposição da fase ferrita, que

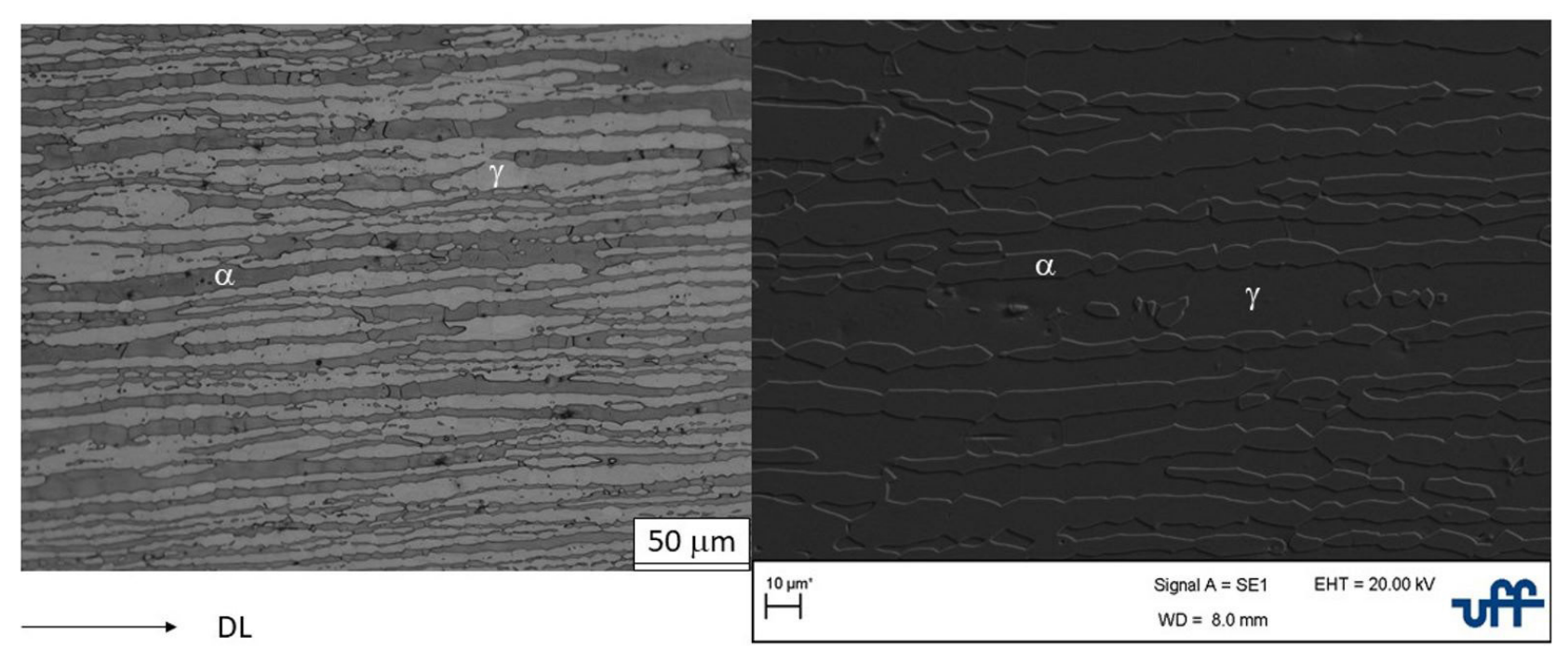

(a)

(b)

Figura 4. Micrografia da amostra como recebida (Direção de laminação (DL); Ataque Behara; (a) Microscopia Ótica - fase ferrita $\alpha$ : fase escura; fase austenita $\gamma$ : fase clara; (b) Microscopia eletrônica de varredura - Elétrons secundários (SEI). 
Tabela 2. Composição química das fases na amostra como recebida (CR) e da fase sigma $(\sigma)$ e austenita secundária $\left(\gamma_{2}\right)$ nas amostras após resfriamento controlado (Resf $\sigma$ e Resf $\gamma_{2}$ )

\begin{tabular}{cccc}
\hline & $\mathbf{C r}$ & $\mathbf{N i}$ & Mo \\
\hline $\operatorname{CR} \alpha$ & $26,2 \pm 0.5$ & $5,5 \pm 0,2$ & $8,1 \pm 0,3$ \\
$\operatorname{CR} \gamma$ & $23,8 \pm 0,5$ & $8,0 \pm 0,4$ & $4,3 \pm 0,2$ \\
$\operatorname{Resf} \sigma$ & $27,2 \pm 1,0$ & $3,4 \pm 0,2$ & $6,8 \pm 0,9$ \\
$\operatorname{Resf} \gamma_{2}$ & $25,0 \pm 0,5$ & $10,8 \pm 1,0$ & $2,4 \pm 0,4$ \\
\hline
\end{tabular}

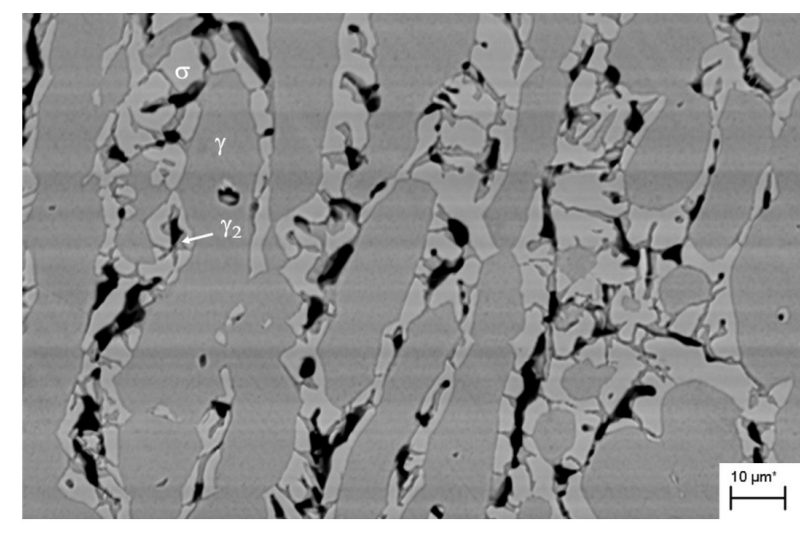

(a)

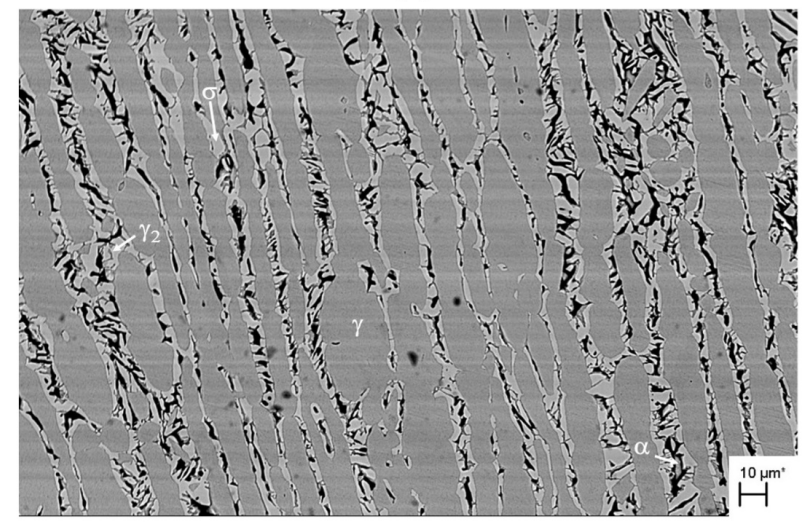

(b)

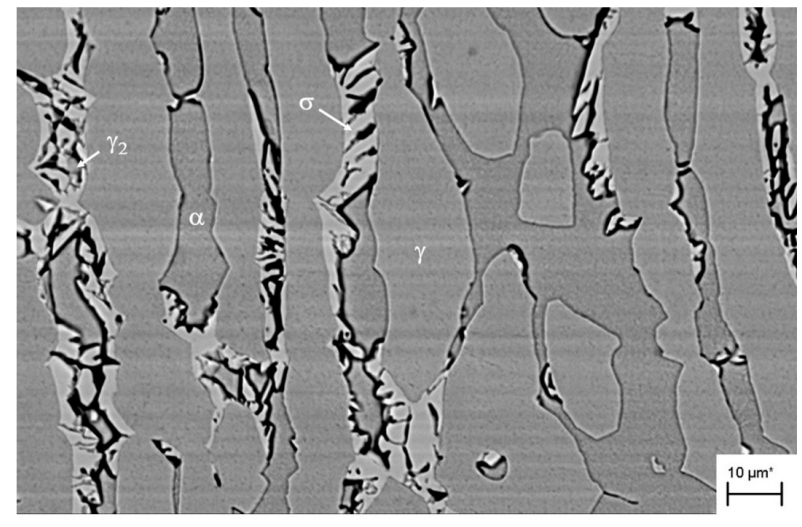

(c)

Figura 5. Micrografias do aço inoxidável superduplex, MEV - Sinal retroespalhado (BSD), (a) taxa I, $0^{\circ} \mathrm{C} / \mathrm{min}$; (b) $2,0^{\circ} \mathrm{C} / \mathrm{min}$; (c) $3,5^{\circ} \mathrm{C} / \mathrm{min}$. Austenita $\gamma$ : coloração cinza claro; fase sigma $\sigma$ : cinza claro que consome a $\alpha$; austenita secundária $\gamma_{2}$ : coloração cinza intercalada com a $\sigma$; partes pretas: desníveis).

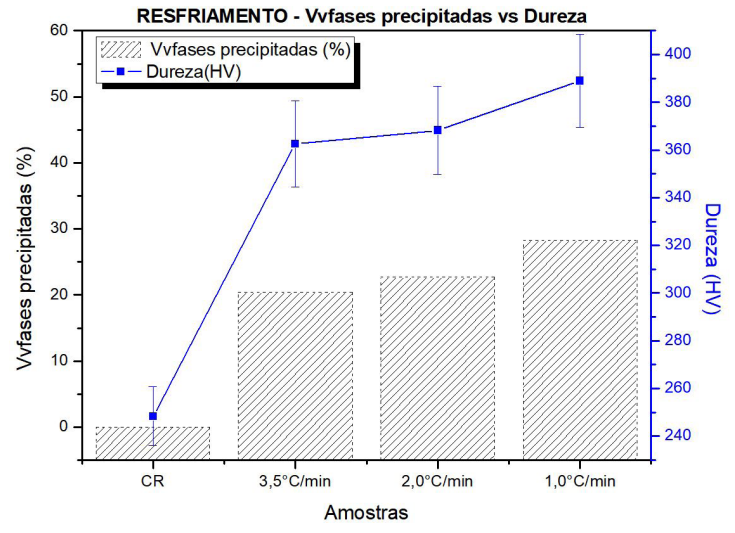

Figura 6. Fração volumétrica das fases precipitadas em relação à dureza nas amostras: $\mathrm{CR}$ e nas taxas de resfriamento $3,5^{\circ} \mathrm{C} / \mathrm{min}$, $2,0^{\circ} \mathrm{C} / \mathrm{min}$ e $1,0^{\circ} \mathrm{C} / \mathrm{min}$.

se dá geralmente através de uma reação eutetóide, formando sigma e austenita secundária. As composições das fases na amostra CR e nas amostras sob resfriamento controlado estão de acordo com as composições apresentadas na literatura. $[1,6,7,9-11,13,17,19,21,22]$

A fase sigma rica em $\mathrm{Cr}$ e Mo, deixa a matriz ferrítica sem os seus principais estabilizadores e a fase austenítica se torna mais estável devido a presença de níquel dissolvido, favorecendo a transformação alotrópica de ferrita para austenita. No caso, austenita secundária. A fração volumétrica de sigma + austenita secundária foi medida para cada taxa de resfriamento e seu resultado foi comparado com o valor de microdureza Vickers para cada amostra, Figura 6. Inicialmente, a dureza apresentada pela amostra foi de 248,4 HV. Nota-se que para a condição de resfriamento contínuo para a taxa de $3,5^{\circ} \mathrm{C} / \mathrm{min}$ com uma fração volumétrica de fases precipitadas igual a 0,204 tem-se a dureza de $362,6 \mathrm{HV}$, para a taxa de $2,0^{\circ} \mathrm{C} / \mathrm{min}$ com uma fração volumétrica de fases precipitadas igual a 0,227 tem-se a dureza de $368,3 \mathrm{HV}$ e para a taxa de $1,0^{\circ} \mathrm{C} / \mathrm{min}$, com uma fração volumétrica de fases precipitadas de 0,288 o valor de dureza foi de $389 \mathrm{HV}$.

A fim de verificar uma relação entre os dados de dureza e a fração volumétrica das fases precipitadas nas amostras, foi realizado um ajuste linear para determinar o coeficiente de determinação $\mathrm{R}^{2}$, conforme Figura 7, cujo valor é 0,98 . Assim, o aumento da dureza está diretamente relacionado com o aumento da porcentagem de fases intermetálicas presentes nas amostras. 


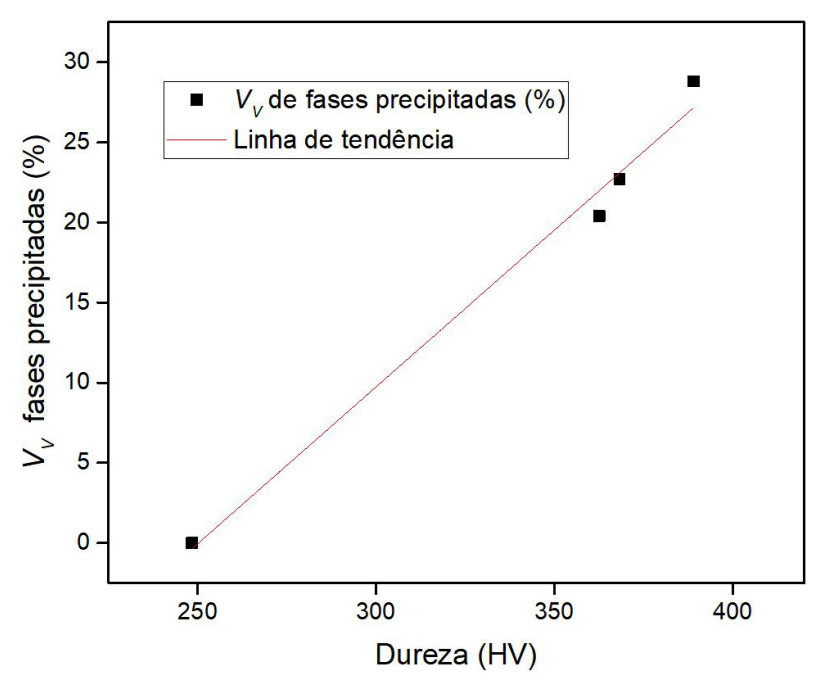

Figura 7. Correlação entre fração volumétrica da fase sigma e a microdureza nas amostras $\mathrm{CR}$ e taxas de resfriamento de $3,5^{\circ} \mathrm{C} / \mathrm{min}$; $2,0^{\circ} \mathrm{C} / \mathrm{min}$ e $1,0^{\circ} \mathrm{C} / \mathrm{min}$.

A partir das micrografias retratadas nas Figuras 5 e dados das frações volumétricas das fases precipitadas, Figura 6 , é possível obter a relação destas taxas de resfriamento com o processo de cinética de formação da fase sigma. Pois o processo de formação é termicamente ativado, onde ocorre a difusão dos elementos $\mathrm{Cr}$ e Mo da ferrita para sigma. Logo, o tempo de exposição do material com relação à temperatura é relevante. Assim com menores taxas de resfriamento $\left(1,0^{\circ} \mathrm{C} / \mathrm{min}\right)$ o material é exposto a altas temperaturas por mais tempo, resultando em maior teor de precipitação das fases intermetálicas. No caso a ferrita vai sendo consumida pelas fases precipitadas.
Com o propósito de avaliar a influência destas fases intermetálicas em relação as propriedades mecânicas, a Figura 7, apresenta a relação de fração volumétrica das fases precipitadas e a dureza para cada taxa de resfriamento. Nota-se que com o aumento da fração volumétrica de sigma, obtém-se uma dureza mais elevada. Isto se deve ao fato dos elementos que compõe a fase sigma, rica em $\mathrm{Cr}$ e Mo, Tabela 2, ao formarem o composto intermetálico tornam o material duro e frágil.

\section{CONCLUSÃO}

A precipitação da fase sigma e austenita secundária no aço inoxidável superduplex $\mathrm{F} 53 \mathrm{em}$ ciclos térmicos de resfriamento controlado foi comprovada mediante os resultados adquiridos da simulação térmica e caracterização microestrutural. As primeiras derivadas dos dados dilatométricos, revelaram picos negativos na faixa de temperatura de formação de precipitados intermetálicos como a fase sigma. Através da análise pela microscopia eletrônica de varredura/EDS foi possível validar o surgimento da fase sigma e austenita secundária. Com a redução da taxa de resfriamento tem-se um aumento da quantidade de sigma presente, o que promove consequentemente um aumento da dureza do material.

\section{Agradecimentos}

Às agências brasileiras de fomento à pesquisa (CNPq, CAPES - código financeiro 00I e FAPERJ) pelo apoio financeiro concedido.

\section{REFERÊNCIAS}

I Fonseca GS, Mendes PSN, Silva ACM. Sigma phase: nucleation and growth. Metals. 2019;9(I):34. http://dx.doi. org/I0.3390/met90I 0034.

2 Fonseca G, Barbosa L, Ferreira E, Xavier C, Castro J. Microstructural, mechanical, and electrochemical analysis of duplex and superduplex stainless steels welded with the autogenous TIG process using different heat input. Metals. 2017;7(I2):538. http://dx.doi.org/10.3390/met7/20538.

3 Fonseca GS, Oliveira PM, Diniz MG, Bubnoff DV, Castro JA. Sigma phase in superduplex stainless steel: formation, kinetics and microstructural path. Materials Research. 2017;20(I):249-255. http://dx.doi.org/10.1590/19805373-mr-2016-0436.

4 Gennari C, Pezzato L, Piva E, Gobbo R, Calliari I. Influence of small amount and different morphology of secondary phases on impact toughness of UNS S32205 Duplex Stainless Steel. Materials Science and Engineering A. 2018;729:149-156. http://dx.doi.org/10.1016/j.msea.2018.05.063.

5 Santos DC, Magnabosco R. Kinetic study to predict sigma phase formation in duplex stainless steels. Metallurgical and Materials Transactions. A, Physical Metallurgy and Materials Science. 20I6;47(4):I554-I565. http://dx.doi. org/I0.1007/s I |66I-016-3323-z.

6 Sathirachinda N, Pettersson R, Pan J. Depletion effects at phase boundaries in 2205 duplex stainless steel characterized with SKPFM and TEM/EDS. Corrosion Science. 2009;5 I(8): I850-1860. http://dx.doi.org/I0. I0I6/j. corsci.2009.05.012. 
7 Escriba DM, Materna-Morris E, Plaut RL, Padilha AF. Chi-phase precipitation in a duplex stainless steel. Materials Characterization. 2009;60(I I): I2 |4-12 19. http://dx.doi.org/I0.1016/j.matchar.2009.04.013.

8 Magnabosco R. Kinetics of sigma phase formation in a duplex stainless steel 2. Experimental procedure. Materials Research. 2009; I2(3):32 I-327. http://dx.doi.org/I0.I590/SI5 I6-I43920090003000I2.

9 Calliari I, Zanesco M, Ramous E. Influence of isothermal aging on secondary phases precipitation and toughness of a duplex stainless steel SAF 2205. Journal of Materials Science. 2006;4I (22):7643-7649. http://dx.doi.org/I0.I007/ sl0853-006-0857-2.

10 Dobranszky J, Szabo PJ, Berecz T, Hrotko V, Portko M. Energy-dispersive spectroscopy and electron backscatter diffraction analysis of isothermally aged SAF 2507 type superduplex stainless steel. Spectrochimica Acta. Part B, Atomic Spectroscopy. 2004;59(I0-I I): I 78I - I 788. http://dx.doi.org/ I 0. I0 I6/j.sab.2004.07.0 I0.

I I Villanueva DME, Junior FCP, Plaut RL, Padilha AF. Comparative study on sigma phase precipitation of three types of stainless steels: austenitic, superferritic and duplex. Materials Science and Technology. 2006;22(9): I 098- I I04. http:// dx.doi.org/I0. I I 79/1 74328406X109230.

I 2 Elmer JW, Palmer TA, Specht ED. Direct observations of sigma phase formation in duplex stainless steels using In-situ synchrotron X-ray diffraction. Metallurgical and Materials Transactions. A, Physical Metallurgy and Materials Science. 2007;38(3):464-475. http://dx.doi.org/I0. I007/s I I66 I-006-9076-3.

I 3 Badji R, Bouabdallah M, Bacroix B, Kahloun C, Belkessa B, Maza H. Phase transformation and mechanical behavior in annealed 2205 duplex stainless steel welds. Materials Characterization. 2008;59(4):447-453. http://dx.doi. org/10.1016/j.matchar.2007.03.004.

I4 Fan K, Liu F, Ma YZ, Yang GC, Zhou YH. Modeling of -phase precipitation in a 2205 duplex stainless steel using an analytical soft impingement treatment. Materials Science and Engineering A. 20 I 0;527( I 8- I9):4550-4553. http:// dx.doi.org/10.1016/j.msea.2010.04.074.

I5 Padilha AF, Rios PR. Decomposition of austenite in austenitic stainless steels. ISIJ International. 2002;42(4):325-327. http://dx.doi.org/I0.2355/isijinternational.42.325.

I6 Mittemeijer EJ, Cheng L, Schaaf PJ, Brakman CM, Korevaar BM. Analysis of nonisothermal transformation kinetics; tempering of iron-carbon and iron-nitrogen martensites. Metallurgical Transactions. A, Physical Metallurgy and Materials Science. 1988; 19. http://dx.doi.org/10.1007/bf02628377.

$17 \mathrm{Kim}$ Y. Phase transformation in cast duplex stainless steels [thesis]. Ames: lowa State University; 2004.

I8 Kim YJ, Chumbley LS, Gleeson B. Continuous cooling transformation in cast duplex stainless steels CD3MN and CD3MWCuN. Journal of Materials Engineering and Performance. 2008; I 7(2):234-239. http://dx.doi.org//0.1007/ sl |665-007-9|34-z.

19 Rivolta B, Gerosa R, Tavasci F. The dilatometric technique for studying sigma phase precipitation kinetics in F55 steel grade. Journal of Thermal Analysis and Calorimetry. 2018; I32(2):869-877. http://dx.doi.org/I0. I007/s I0973-0I76940-x.

20 Russ JC, Dehoff RT. Practical stereology. 2nd ed., New York: Kluwer Academic/Plenum Publishers; 2000. http:// dx.doi.org/10.1007/978-I-46/5-1233-2.

2I Londoño AJR. Estudo da precipitação de nitreto de cromo e fase sigma por simulação térmica da zona afetada pelo calor na soldagem multipasse de aços inoxidáveis duplex [dissertação]. São Paulo: Universidade de São Paulo; 1997.

22 Lopes JTB. Influência da presença de fases frágeis e da temperatura nas propriedades de propagação de trinca por fadiga do aço inoxidável duplex UNS S3 1803 [tese]. Campinas: Universidade Estadual de Campinas; 2006.

Recebido em: 30 Nov. 2018

Aceito em: 23 Ago. 2019 